\title{
Embarazadas con lupus eritematoso sistémico en el hospital Clínico Regional de Concepción, Chile
}

\author{
Susana Sabat A. ${ }^{a}$, Michelle Vinet M.a , Camila Sanhueza F.a , Andrea Galdames G. ${ }^{a}$, \\ Irene Castro E. ${ }^{1}$, Andrés Caballero . $^{2}$ \\ ${ }^{1}$ Servicio de Reumatología, ${ }^{2}$ Servicio de Ginecología y Obstetricia. Hospital Clínico Regional de Concepción, Chile. \\ a Interna, Escuela de Medicina, Universidad de Concepción, Chile.
}

\section{RESUMEN}

Antecedentes: El lupus eritematoso sistémico (LES) afecta principalmente a mujeres en edad fértil. El embarazo en estas pacientes puede asociarse con múltiples complicaciones. Objetivo: Caracterizar a las embarazadas con LES durante 10 años en el Hospital Clínico Regional de Concepción. Métodos: Se realizó un estudio descriptivo retrospectivo que consistió en la revisión de fichas clínicas. Se analizaron las variables: edad, años de enfermedad desde el diagnóstico, historia obstétrica, presencia de reactivaciones, anticuerpos maternos y complicaciones materno-fetales. Resultados: Durante el periodo de estudio hubo $49 \mathrm{em}-$ barazos en 21 pacientes con LES. El 12,2\% terminó en aborto, un $2 \%$ en óbitos, y un total de 43 nacidos vivos. La edad promedio de las pacientes al momento del diagnóstico de LES fue 24,5 años. El $67 \%$ fueron diagnosticadas antes de su primer embarazo. En el total de pacientes el 85,7\% presentaron ANA positivo, $57,1 \%$ antiDNA positivo, $52,4 \%$ aRo positivo y $33,3 \%$ aLa positivo. En los caso de abortos, aRo y aLa se encontraban positivos en $66,7 \%$. Las anticardiolipinas se encontraban alteradas en $33,3 \%$ de los abortos. Durante el embarazo el $32,6 \%$ tenía LES activo y $34,7 \%$ en el postparto. El $53,5 \%$ de los recién nacidos no tuvieron complicaciones. La complicación más frecuente fue la prematuridad con $55 \%$. La mortalidad perinatal de la serie fue de 46,5/1000 nacidos vivos (2/43). No hubo muertes maternas. Conclusión: Es importante la educación respecto al embarazo en pacientes con LES. Debemos resaltar en promover que estas pacientes planifiquen el embarazo en periodo de inactividad, y con controles frecuentes para pesquisar precozmente cualquier complicación.

\section{PALABRAS CLAVES: Lupus eritematoso sistémico, embarazo, complicaciones materno-fetal}

\section{SUMMARY}

Background: The systemic lupus erythematosus (SLE) affects mainly fertile age women. Pregnancy in these patients can associate with multiple complications. Aims: To characterize the pregnant women with SLE during 10 years in the Hospital Clínico Regional de Concepción, Chile. Methods: We made a retrospective descriptive study which consisted in clinical files revision. The following variables were analyzed: age, years with disease since diagnose, obstetric history, history of reactivation, maternal antibodies and mother-fetus complications. Results: During the time of study there were 49 pregnancies on 21 patients with SLE; $12.2 \%$ ended in abortion, $2 \%$ in late fetal death giving a total of 43 living newborn. The average age of these patients at the moment of diagnose of LES was 24.5 years old; $67 \%$ were diagnosed before their first pregnancy. From the total of patients, $85.7 \%$ presented positive ANA, $57.1 \%$ positive antiDNA, positive aRo in $52.4 \%$ and positive aLa in $33.3 \%$. In case of abortions, aRo and aLa were positive in $66.7 \%$. Anticardiolipins were 
altered in $33.3 \%$ of abortions. During pregnancy $32.6 \%$ had active SLE, and $34.7 \%$ post-partum. Among the newborn, $53.5 \%$ did not have any complications. The most frequent complication was prematurity with a $55 \%$. The perinatal mortality was $46.5 / 1000$ lives births (2/43). There were no maternal deaths. Conclusion: It is important to educate about pregnancy in SLE patients. We must emphasize to promote in those patients a planned pregnancy in inactive period and with frequent controls for early diagnose of any complication.

\section{KEY WORDS: Systemic lupus erythematosus, pregnancy, fetal-maternal complication}

\section{INTRODUCCIÓN}

El lupus eritematoso sistémico (LES) es una enfermedad autoinmune, crónica, multisistémica de evolución y clínica variable, dependiendo de los órganos que se encuentren afectados y de la diversa gama de autoanticuerpos que se producen, con periodos de remisión y reactivación. Afecta principalmente a mujeres en edad fértil, las que tienen tasas de fecundidad similares a las pacientes sin LES (1).

El embarazo en pacientes con esta enfermedad puede asociarse con variadas complicaciones tales como mayor riesgo de aborto, prematurez, retardo del crecimiento intrauterino, preclampsia y lupus neonatal (2). En la embarazada se ha reportado mayor tasa de infecciones, anemia, trombocitopenia, tromboembolismo pulmonar, trombosis venosa profunda, accidente vascular cerebral y muerte (3).

Aún es controversial si hay o no un aumento del riesgo de reactivación de la enfermedad durante el embarazo. Se ha reportado una tasa de reactivación que varía entre el $7-33 \%$, que es similar a la tasa reportada en pacientes no embarazadas con LES (4).

En obstetricia tiene una incidencia de 1:1600 a 3.000 partos (5). El embarazo en pacientes con LES ha sido una opción viable en las últimas décadas debido al desarrollo de tratamientos que han mejorado el control de esta enfermedad y permitir que el embarazo tenga mayores tasas de éxito (6). El pronóstico para la madre y el niño es mejor cuando la enfermedad se encuentra en remisión por lo menos 6 meses antes de ocurrido el embarazo, por lo tanto la anticoncepción y la evaluación preconcepcional son especialmente importantes para las mujeres con esta patología (7). La salud materna y el desarrollo fetal deben monitorizarse frecuentemente durante el embarazo en centros que tengan unidades de alto riesgo obstétrico (8).

El objetivo de nuestro estudio es caracterizar a las pacientes embarazadas con LES en un período de 10 años en el Hospital Clínico Regional de Concepción, Chile.

\section{PACIENTES Y MÉTODOS}

Se realizó un estudio de tipo descriptivo retrospectivo, que consistió en la revisión de fichas de pacientes embarazadas con LES atendidas en el po- liclínico de alto riesgo obstétrico (ARO) durante el periodo comprendido entre los años 2001 - 2011, en el Hospital Clínico Regional de Concepción (HCRC), Chile. Los criterios de inclusión fueron: pacientes embarazadas con LES, rango de edad de 15 a 45 años, información completa en ficha clínica, sin comorbilidad grave no asociada a LES y consentimiento informado para realizar el estudio. Se excluyeron a 3 pacientes por presentar información incompleta en su ficha clínica. Se analizaron las variables edad, años de enfermedad desde el diagnóstico, historia obstétrica, presencia de reactivaciones, anticuerpos maternos y complicaciones materno-fetales. Este estudio fue aprobado por el comité de ética HCRC.

El protocolo de manejo de las pacientes embarazadas con diagnóstico de LES en control en el HCRC depende de la existencia de complicaciones tanto para la madre y feto debido al LES, de la presencia de Rho o La positivos y de la presencia de alteraciones renales.

El protocolo general de manejo contempla en primer lugar la realización de ecografía obstétrica entre las 11-14 semanas para evaluar malformaciones mayores y riesgo de aneuploidias, entre las 22-24 semanas para evaluar la función placentaria, anatomía fetal y riesgo de parto prematuro y entre las 32-34 semanas para evaluar crecimiento fetal y líquido amniótico. En segundo lugar se realiza, desde el comienzo del embarazo, pesquisa de la presencia de anti Rho o La; frente a la positividad de estos, se realiza una evaluación cardiaca fetal periódica a través de monitoreo cardiaco fetal y ecocardiograma fetal hasta el parto. Como tercer punto el manejo incluye en un principio la búsqueda del síndrome antifosfolípidos, que de estar presente se realiza el tratamiento correspondiente. Un cuarto punto incluye la evaluación desde un comienzo la función renal con seguimiento mensual de estos parámetros; a partir de las 24 semanas se solicita un estudio consistente en medición de ácidoúrico y proteinuria de 24 horas para evaluar función renal y presencia de preeclampsia, que en caso de ser negativo es evaluado de forma mensual.

En relación a la interrupción del embarazo, este se efectúa según las complicaciones de su patología de base o frente a la presencia de preeclampsia, síndrome antifosfolípidos, historia obstétrica, etc. En 
caso de no presentar complicaciones maternas ni fetales, el embarazo llega a término con parto vaginal.

\section{RESULTADOS}

Se registraron 21 pacientes que presentaban diagnóstico de LES, quienes durante sus embarazos fueron controladas en el policlínico de Alto Riesgo Obstétrico (ARO), haciendo un total de 49 embarazos durante el período de estudio. Del total de embarazos, $12,2 \%(n=6)$ terminó en abortos y $2 \%(n=1)$ de óbitos, dando un total de 43 nacidos vivos. Hubo un $2 \%(n=1)$ de embarazos gemelares, uno de ellos falleció antes de las 20 semanas de gestación. De los nacidos vivos, el 2,3\% $(n=1)$ falleció en el periodo neonatal. Un 19\% $(n=4)$ de las pacientes presentaban más de un aborto en su historia obstétrica. La edad promedio de las pacientes al momento del diagnóstico de LES fue 24,5 años y el promedio de edad actual fue 34,3 años. No hubo muertes maternas.

Los años de evolución de la enfermedad, según el total de pacientes $(n=21)$ fue: $9,5 \%(n=2)$ menos de 5 años de evolución, el $47,6 \%(n=10)$ entre 5 y 10 años, el 42,8\% (n=9) más de 10 años. En el $67 \%(n=14)$ de las pacientes con LES, la enfermedad fue diagnosticada antes del primer embarazo, y con posterioridad el $33 \%(n=7)$.

Del total de las pacientes que fueron diagnosticadas de LES después del primer embarazo, el $71,4 \%(n=5)$ tuvieron 1 embarazo antes de su diagnóstico de LES, el 14,3\% $(n=1)$ tuvo 2 embarazos, ninguna tuvo 3 embarazos y el 14,3\% $(n=1)$ tuvo 4 embarazos, con un total de 11 embarazos previos al diagnóstico, de los cuales el $63,6 \%(n=7)$ presentó complicaciones maternas, correspondiendo un $28,6 \%(n=2)$ a síndrome hipertensivo del embarazo, al igual que a colestasia intrahepática del embarazo, y el $36,4 \%(n=6)$ complicaciones del neonato, predominando la prematuridad con un $66,7 \%(n=4)$.

En la Tabla I se presenta la distribución por edad al momento del embarazo. El 40,8\% $(n=20)$ de las pacientes se encuentra en el rango de 25 a 29 años.

En la Figura 1 se presenta la presencia de anticuerpos positivos/negativos o complemento alterado, del total de pacientes $(n=21)$. El $85,7 \%(n=18)$ presentan ANA positivo; $57,1 \%(n=12)$ antiDNA positivo; $52,4 \%(n=11)$ aRo positivo y $33,3 \%(n=7)$ aLa positivo.

\section{Tabla I}

DISTRIBUCIÓN POR EDADES DE LAS PACIENTES AL MOMENTO DEL EMBARAZO

\begin{tabular}{ccc}
\hline Edad materna (años) & $\mathrm{n}$ & $\%$ \\
\hline$<15$ & 0 & 0 \\
$15-19$ & 10 & 20,4 \\
$20-24$ & 10 & 20,4 \\
$25-29$ & 20 & 40,8 \\
$30-34$ & 5 & 10,2 \\
$\geq 35$ & 4 & 8,2 \\
Total & 49 & 100 \\
\hline
\end{tabular}

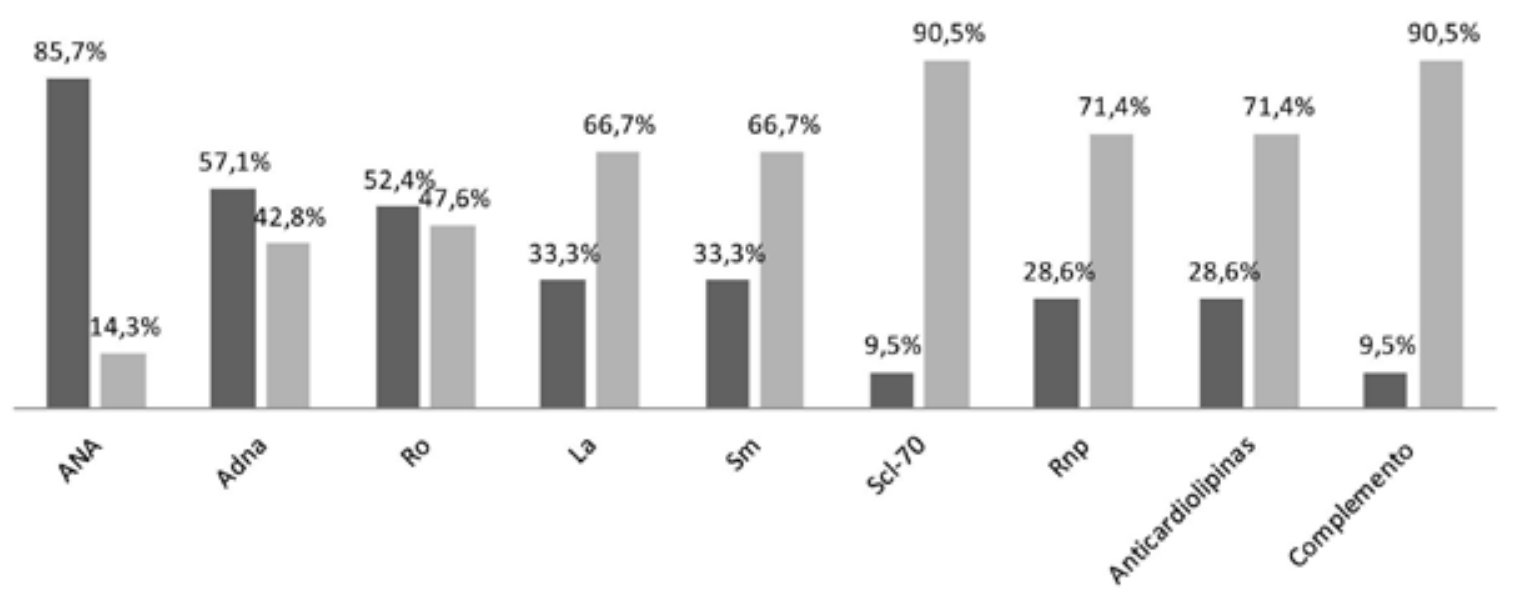

Resultados de exámenes de anticuerpos y complemento

Figura 1. Distribución de positividad o negatividad de los anticuerpos y complemento del total de pacientes $(n=21)$. 
En los casos de óbitos, aRo y aLa estaban positivos. En los casos de abortos, aRo y aLa se encontraban positivos en $66,7 \%(n=4)$ cada uno. Las anticardiolipinas estaban alteradas en el $33,3 \%(n=2)$ de los abortos.

Durante el embarazo el 32,6\% $(n=16)$ de las pacientes estaba con enfermedad activa y el $34,7 \%$ $(n=17)$ en el postparto. Del total de embarazos con enfermedad activa en el embarazo el $62,5 \%(n=10)$ presentaron complicaciones maternas, correspondiendo el $60 \%(n=6)$ a abortos, y un $62,5 \%(n=10)$ presentó complicaciones del neonato.

Según tipo de parto, el 53\% $(n=23)$ fue vía vaginal,y $47 \%(n=20)$ por cesárea.

La edad gestacional de los nacidos vivos se detalla en la Tabla II, el $81,4 \%(n=35)$ del total de los nacidos vivos, tenían al nacer entre las 35 y 40 semanas. El peso promedio de los recién nacidos fue de 2854 gramos. Según la relación peso y edad gestacional: el $6,7 \%(n=2)$ de los recién nacidos fue clasificado como pequeño para la edad gestacional (percentil <10), el 79,1\% ( $n=34)$ como adecuado para la edad gestacional (percentil 10-90), y el $4,6 \%(n=2)$ como grande para la edad gestacional (percentil >90).

Las complicaciones en el embarazo se presentaron en el $45 \%(n=22)$ de estos, existiendo en algunos casos más de una complicación (Tabla III). De todos los embarazos, un $20 \%(n=6)$ presentó aborto, un $3,3 \%(n=1)$ óbito y un $10 \%(n=3)$ trombopenia. Otras complicaciones fueron oligohidroamnios, polihidroamnios y restricción del crecimiento intrauterino.

En los recién nacidos, el 53,5\% $(n=23)$ no tuvieron complicaciones en su periodo neonatal. Los casos en que si existieron complicaciones, se detallan en la Figura 2, donde se describe un total de 25 complicaciones sumando las existentes en el total de embarazos, dando este valor por presentarse más de una complicación en algunos casos, siendo la complicación más frecuente la prematuridad con $44 \%(n=11)$ del total de recién nacidos con complicaciones.

Tabla II

DISTRIBUCIÓN POR EDAD GESTACIONAL DE LOS NACIDOS VIVOS

\begin{tabular}{ccc}
\hline Edad gestacional (semanas) & $\mathrm{n}$ & $\%$ \\
\hline$<20$ & 1 & 2,3 \\
$20-24$ & 0 & 0 \\
$25-29$ & 1 & 2,3 \\
$30-34$ & 5 & 11,7 \\
$35-39$ & 35 & 81,3 \\
$\geq 40$ & 1 & 2,3 \\
Total & 43 & 100 \\
\hline
\end{tabular}

La tasa de mortalidad perinatal fue de 46,5 por mil nacidos vivos (2/43).

\section{DISCUSIÓN}

Actualmente las mujeres con LES presentan una mejor calidad y expectativa de vida, lo que también ha incrementado el número de embarazos en estas pacientes. Aunque en la mayoría de los casos los embarazos logran llegar a término, en este tipo de pacientes se mantiene una condición de alto riesgo. Pacientes con LES tienen un mayor riesgo de complicaciones durante el embarazo, en nuestra serie hubo una tasa de mortalidad perinatal de 46,5 por mil nacidos vivos. En nuestro trabajo un $45 \%$ de las pacientes presentaron algún tipo de complicación durante este periodo.

Una de las complicaciones que se ha reducido en los últimos 40 años es el aborto, en 1960-1965 la incidencia de abortos llegaba a un $43 \%$ descendiendo al $17 \%$ en el trienio $2000-2003$, en nuestro trabajo el aborto tuvo una prevalencia del $12 \%$, cifras similares a las que se han descrito en la literatura (9).

\section{Tabla III \\ DISTRIBUCIÓN DE LAS COMPLICACIONES DURANTE EL EMBARAZO, DEL TOTAL DE EMBARAZOS}

\begin{tabular}{lcc}
\hline Complicación del embarazo & $\mathrm{n}$ & $\%$ \\
\hline Hepatitis lúpica & 1 & 3,3 \\
Aborto & 6 & 20 \\
Óbito & 1 & 3,3 \\
Colestasia intrahepática del & 1 & 3,3 \\
embarazo & & \\
Síndrome de HELLP & 2 & 6,7 \\
Arritmia fetal & 1 & 3,3 \\
Trombopenia & 3 & 10 \\
Taquicardia supraventricular materna & 1 & 3,3 \\
Oligohidroamnios & 1 & 3,3 \\
Polihidroamnios & 1 & 3,3 \\
Síndrome hipertensivo del embarazo & 3 & 10 \\
Edema pulmonar agudo & 1 & 3,3 \\
Restricción del crecimiento & 2 & 6,7 \\
intrauterino & & \\
Desprendimiento prematuro de & 1 & 3,3 \\
placenta normoinserta & & \\
Sufrimiento fetal agudo & 2 & 6,7 \\
Rotura prematura de membrana & 2 & 6,7 \\
Trombosis venosa profunda & 1 & 3,3 \\
Total & 30 & 100 \\
\hline & & \\
\hline & &
\end{tabular}




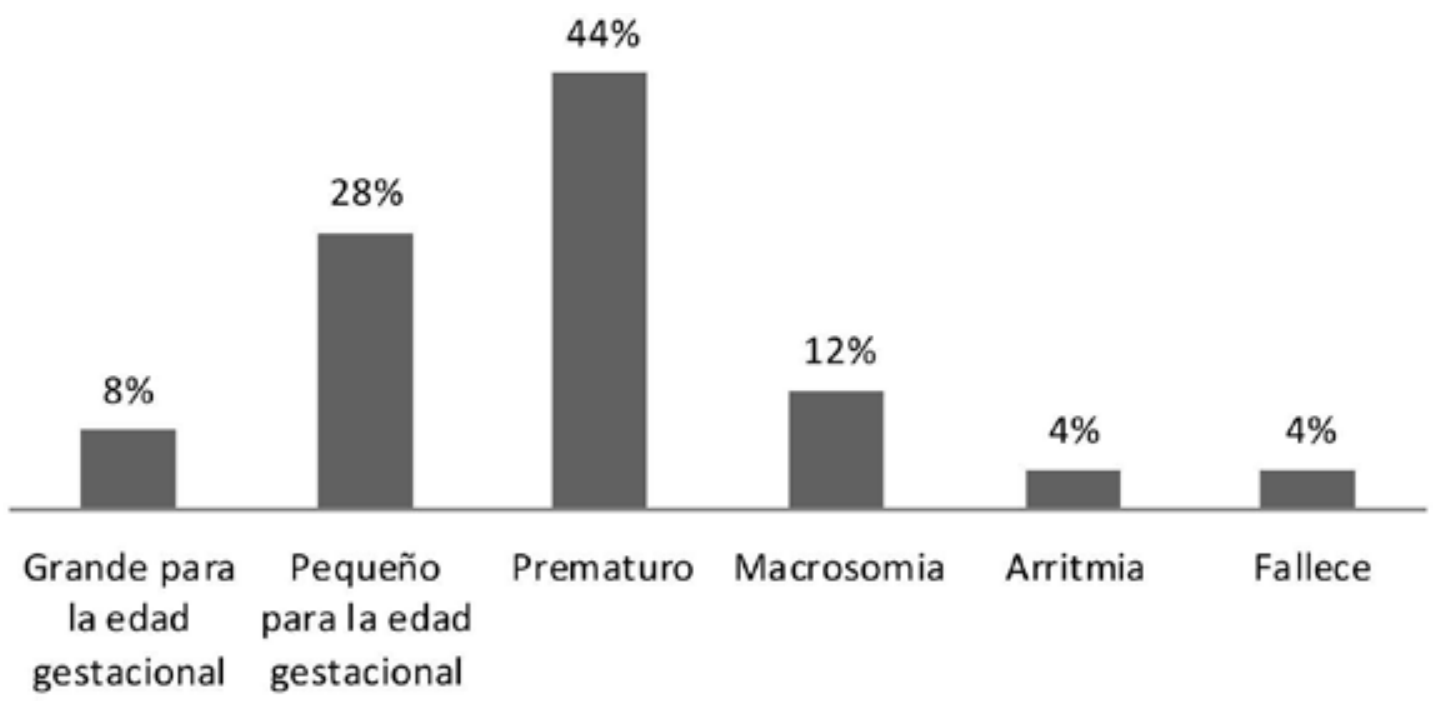

Figura 2. Complicaciones neonatales.

La preeclampsia en embarazos con LES varía del $5 \%$ al $38 \%$ en diversas series publicadas, porcentaje más alto que el de los embarazos en mujeres sin lupus (10), donde se observa entre un 3 y $14 \%$ de los embarazos. Puede ocurrir incluso con mayor frecuencia entre las mujeres con enfermedad renal, el síndrome antifosfolípidos (SAF), la diabetes mellitus, o episodio previo de preeclampsia (11), en nuestro centro el porcentaje de las pacientes con LES que desarrollaron el síndrome hipertensivo del embarazo fue de $6 \%$, mientras que el síndrome de HELLP se presentó en el $4 \%$ de ellas. Otras complicaciones como la hepatitis lúpica, el desprendimiento prematuro de placenta normoinserta, la trombosis venosa profunda, el óbito fetal, la arritmia fetal, la colestasia intrahepática del embarazo, el edema pulmonar agudo, el oligo y polihidroamnios se presentaron en un porcentaje mínimo de pacientes lo que podríamos atribuirlo a un buen control previo y durante el embarazo de nuestras pacientes con lupus, pero también podría deberse a la baja casuística de nuestro estudio. A pesar de las complicaciones presentadas por las pacientes, no hubo muertes maternas durante este estudio.

La presión arterial, proteinuria, clearence de creatinina, concentraciones de complemento, los títulos de anti-DNA y el hemograma son obtenidos al diagnóstico del embarazo, realizándose seguimiento con algunos de estos exámenes según la evolución del embarazo y la presencia de alteraciones en los exámenes iniciales (12). El control del bienestar fetal es realizado a través de ecografía obstétrica en las semanas anteriormente señaladas, aumentando la frecuencia de estas frente a hallazgos patológicos (13). En caso de la presencia de anti Rho o La positivos, se realiza evaluación cardiaca fetal cada dos semanas y ecocardiograma fetal a las 2224 semanas, ambas hasta el parto. En nuestra serie no hubo complicaciones cardiacas fetales.

La actividad del LES puede incrementarse durante el embarazo en el período inmediatamente posterior al parto. La tasa de activación de la enfermedad van desde $13,5 \%$ hasta $65 \%$ de los embarazos (14), sin embargo la incidencia de activación con el embarazo ha disminuido progresivamente en las últimas décadas, sobre todo en aquellos en remisión en el comienzo del embarazo; la frecuencia es mayor en pacientes con enfermedad activa en el momento de la concepción $(15,16)$. En nuestro caso $32 \%$ de las pacientes se presentaron con LES activo durante el embarazo, mientras que el $34,7 \%$ de ellas presentó LES activo durante el postparto.

En cuanto a las pruebas de laboratorio los anticuerpos antiDNA son una prueba de diagnóstico altamente sensible y específico para LES. Un resultado positivo de anti-DNA en el segundo trimestre se asocia con una mayor tasa de pérdida del embarazo y de parto prematuro (3), el $25 \%$ de las pacientes de este estudio presentó anticuerpos antiDNA (+) durante el embarazo. La activación del complemento es una manifestación de exacerbación del lupus. La hipocomplementemia se presentó en $4 \%$ de ellas. Sin embargo durante el embarazo normal, hay un aumento de $10-50 \%$ en el nivel de los componentes del complemento, probablemente como 
resultado del estrógeno impulsando un aumento de la síntesis hepática (17), por lo que se debe tener en consideración al analizar los resultados.

Es necesario destacar que si bien el $85,7 \%$ de nuestras pacientes $(n=18)$ tenían ANA $(+)$, este es un examen sólo válido para el diagnóstico (18) y no es necesario repetirlo durante la vida de una paciente diagnosticada y menos durante el embarazo, ya que no mide actividad y solo estaremos utilizando recursos que no nos brindan utilidad.

Al revisar la literatura chilena sobre el tema nos encontramos con un solo estudio similar al nuestro (5), realizado en Valdivia, con periodo de tiempo observado similar ( 9 años versus 10 años en nuestro estudio), pero llama la atención que luego del diagnóstico de LES solo el $41 \%(n=7)$ de las pacientes tuvieron un parto y todas ellas presentaban un lupus inactivo por un periodo de al menos 6 meses. Resulta importante especificar que la mayoría de las pacientes de Valdivia no poseían características de mayor riesgo obstétrico (LES con compromiso renal, síndrome antifosfolípidos $2^{\circ}$, anticuerpos anti Ro/La, LES activo al inicio del embarazo y mala historia obstétrica) lo que podría explicar la ausencia de complicaciones que presentó dicho estudio.

Por todo lo anterior es que se debe hacer hincapié en la planificación responsable de un embarazo planificado, idealmente en una paciente con LES inactivo y con controles frecuentes para pesquisar las posibles complicaciones materno-perinatales $(19,20)$.

\section{CONCLUSIÓN}

LES es una condición de alto riesgo durante el embarazo y que pueden afectar de igual manera a la madre como al feto, con una prevalencia superior a la encontrada en embarazadas sin esta enfermedad. Es importante la educación, sobre todo con respecto al tema del embarazo, ya que la mayoría de las pacientes son diagnosticadas en rangos etarios fértiles y presentarán deseos de embarazo alguna vez en su vida. Por lo anterior es que se enfatiza la planificación responsable de un embarazo, idealmente en pacientes con LES inactivo y con controles frecuentes para pesquisar las posibles complicaciones materno-perinatales.

\section{REFERENCIAS}

1. Ruiz-Irastorza G, Lima F, Alvs J, Khamashta MA, Simpson J, Hughes GR, Buchanan NM. Increased rate of lupus flare during pregnancy and the puerperium: A prospective study of 78 pregnancies. $\mathrm{Br} \mathrm{J}$ Rheumatol 1996;35:133-8.

2. Teixeira $V$, Gonçalo M. Neonatal lupus erythematosus
- review of pathophysiology and clinical implications. Acta Reumatol Port 2012;37:314-23.

3. Clowse $M$, Jamison M, Myers E, James A. A national study of the complications of lupus in pregnancy. Am J Obstet Gynecol 2008;199:127.e1-127.e6.

4. Hayslett JP. Maternal and fetal complications in pregnant women with systemic lupus erythematosus. Am J Kidne yDis 1991;17:123-6.

5. Gutiérrez R, Caro J, Pérez L, Ramírez J. Lupus eritematoso sistémico y embarazo. Rev Chil Obstet Ginecol 2002;67:121-4.

6. Clark CA, Spitzer KA, Laskin CA. Decrease in pregnancy loss rates in patients with systemic lupus erythematosus over a 40-year period. J Rheumatol 2005;32:1709-12.

7. Urowitz M, Gladman DD, Farewell VT, Stewart J, McDonald J. Lupus and pregnancy studies. Arthritis Rheumat 1993;36:1392-97.

8. Mintz G, Niz J, Gutierrez G, Garcia-Alonso A, Karchmer $S$. Prospective study of pregnancy in systemic lupus erythematosus: results of a multidisciplinary approach. J Rheumatol 1986;13:732-9.

9. Clark CA, Spitzer KA, Nadler JN, Laskin CA. Preterm deliveries in women with systemic lupus erythematosus. J Rheumatol 2003;30:2127-32.

10. Kitridou RC. The mother in systemic lupus erythematosus. In: Wallace DJ, Hahn BH, eds. Dubois' lupus erythematosus .5th Ed. Baltimore: Williams \& Wilkins, 1997: 967-1002.

11. Repke JT. Hypertensive disorders of pregnancy. Differentiating preeclampsia from active systemic lupus erythematosus. J Reprod Med 1998;43:350-4.

12. Mok CC, Wong RW. Pregnancy in systemic lupus erythematosus. Postgrad Med J 2001;77:157-65.

13. Madazli R, Bulut B, Erenel H, Gezer A, Guralp O. Systemic lupus erythematosus and pregnancy. J Obstet Gynaecol 2010;30:17-20.

14. Stojan G, Baer AN. Flares of systemic lupus erythematosus during pregnancy and the puerperium: prevention, diagnosis and management. Expert Rev Clin Immunol 2012;8:439-53.

15. Liu J, Zhao Y, Song Y, Zhang W, Bian X, Yang J, et al. Pregnancy in women with systemic lupus erythematosus: a retrospective study of 111 pregnancies in Chinese women. $\mathrm{J}$ Matern Fetal Neonatal Med 2012;25:261-6.

16. Lockshin MD, Reinitz E, Druzin ML, Murrman M, Estes D. Lupus pregnancy: case control prospective study demonstrating absence of lupus exacerbation during or after pregnancy. Am J Med 1984;77:893-898.

17. Buyon JP, Kalunian KC, Ramsey-Goldman R, Petri MA, Lockshin MD, Ruiz-Irastorza G, et al. Assessing disease activity in SLE patients during pregnancy. Lupus 1999;8:677-84.

18. Javier-Zepeda CA. Anticuerpos anti-nucleares. Una familia diversa. Rev Med Hond 2002;70:189-93.

19. Lateef A, Petri M. Managing lupus patients during pregnancy. Best Pract Res Clin Rheumatol 2013;27:435-47.

20. Ostensen M, Clowse M. Pathogenesis of pregnancy complications in systemic lupus erythematosus. Curr Opin Rheumatol 2013;25:591-6. 